\title{
Cost-Utility Analysis of Molecular Testing for Tuberculosis Diagnosis in Suspected Pulmonary Tuberculosis in Thailand
}

\author{
Natthakan Chitpim', Jiraphun Jittikoon ${ }^{2}$, Wanvisa Udomsinprasert ${ }^{2}$, Surakameth Mahasirimongkol ${ }^{3}$, \\ Usa Chaikledkaew ${ }^{4,5}$
}

'Social, Economic and Administrative Pharmacy (SEAP) Graduate Program, Faculty of Pharmacy, Mahidol University, Bangkok, Thailand; ${ }^{2}$ Department of Biochemistry, Faculty of Pharmacy, Mahidol University, Bangkok, Thailand; ${ }^{3}$ Medical Genetics Center, Medical Life Sciences Institute, Department of Medical Sciences, Ministry of Public Health, Bangkok, Thailand; ${ }^{4}$ Social Administrative Pharmacy Division, Department of Pharmacy, Faculty of Pharmacy, Mahidol University, Bangkok, Thailand; ${ }^{5}$ Mahidol University Health Technology Assessment (MUHTA) Graduate Program, Mahidol University, Bangkok, Thailand

Correspondence: Usa Chaikledkaew, Social and Administrative Pharmacy Division, Department of Pharmacy, Faculty of Pharmacy, Mahidol University, 447 Sri-Ayudhaya Road, Rajathevi, Bangkok, 10400, Thailand, Tel +66 2-644-8679 ext 53 I7, Fax +66 2-644-8694, Email usa.chi@mahidol.ac.th

\begin{abstract}
Purpose: Given the lack of economic evaluation study of molecular testing in Thailand, this study aimed to evaluate the cost-utility of molecular testing algorithms including Xpert MTB/RIF and the loop-mediated isothermal amplification (TB-LAMP) in the general population suspected of having pulmonary TB based on a societal perspective.

Methods: A hybrid decision tree Markov model using a 1-month cycle length was used to evaluate costs and outcomes of five TB diagnostic algorithms: 1) sputum smear microscopy (SSM) with culture and drug susceptibility testing (DST), 2) Xpert MTB/RIF addon, 3) Xpert MTB/RIF initial, 4) TB-LAMP add-on, and 5) TB-LAMP initial during a lifetime period. All costs were calculated in 2021 Baht, and results were presented as an incremental cost-effectiveness ratio (ICER) for molecular testing compared with SSM with culture. One-way sensitivity and probability analyses were used to evaluate uncertainty input parameters.

Results: TB-LAMP was less expensive overall (6565 Baht) than Xpert MTB/RIF (7010 Baht) and SSM with culture (6845 Baht). Molecular testing was projected to improve quality adjusted life year (QALY) by 0.53 to 0.94 years. In comparison to SSM with culture and DST, providing an initial TB-LAMP test was the most preferred choice. Xpert MTB/RIF Initial had the lowest ICER (197 Baht per QALY gained), followed by TB-LAMP Add-on (993 Baht per QALY gained) and Xpert MTB/RIF Add-on (3940 Baht per QALY gained). One-way sensitivity analysis uncovered that sensitivity of TB-LAMP was greater than that of other parameters.

Conclusion: Providing molecular testing including Xpert MTB/RIF and TB-LAMP as either initial or add-on test for TB diagnosis was more cost-effective than SSM with culture and DST in the general population with suspected pulmonary TB in Thailand. Our study could provide useful evidence to policymakers advocating for inclusion of molecular testing in the universal health coverage benefit package in Thailand.
\end{abstract}

Keywords: molecular testing, economic evaluation, diagnosis tuberculosis, Xpert MTB/RIF, TB-LAMP

\section{Introduction}

Tuberculosis (TB) is an infectious disease that can spread easily from person to person through the air. TB is caused by bacteria, namely Mycobacterium tuberculosis (MTB), which most often affect the lungs (80\%). ${ }^{1}$ In 2020, there were 10 million TB affected people worldwide, of which $2.6 \%$ were confirmed multidrug-resistant TB (MDR-TB) patients and about 1.5 million cases had died. Only $61 \%$ of the TB patients were reported, and $86 \%$ of them successfully received treatment in 2020. The underreported TB patients and underdiagnosed TB were the main causes of the discrepancy between estimated and reported cases. ${ }^{2}$

Thailand is an upper-middle-income country classified by the World Bank and has been included on high-burden country lists for TB and TB/human immunodeficiency virus (HIV). ${ }^{2}$ In 2020, an estimated TB incidence in Thailand was 
about 105,000 people, in which 1.5\% had MDR-TB and approximately $9.5 \%$ died from TB. The number of reported cases was $83 \%$ and the number of patients who were successfully treated was increased to $85 \%$ in $2020 .^{2}$ Although TB is a curable disease, TB treatment can result in a decrease in patient's quality of life in physical, psychological and social aspects as well as an increase in economic burden to TB patients and their families. ${ }^{3}$

Both the Sustainable Development Goals (SDGs) and the World Health Organization (WHO)'s End TB Strategy are recognized as global targets and milestones for reducing the burden of TB disease. The End TB Strategy's 2030 aims include a 90\% reduction in TB deaths and an $80 \%$ reduction in TB incidence (less than 20 cases per 100,000 population, compared to $2015 .^{4}$ To achieve the goal of reducing TB incidence in Thailand, the rate should be decreased by $12.5 \%$ per year, from 171 cases in 2014 to 88 cases per 100,000 population in 2030. Nevertheless, the recent data showed that TB incidence rate was decreased by only $2.7 \%$ per year during the last decade. An important pillar to achieve Thailand Operational Plan to End Tuberculosis 2017-2021 is to increase patient access to early TB diagnosis via using molecular diagnostic tests for all presumptive TB. ${ }^{5}$

In Thailand, a high-burden TB country, the chest x-ray (CXR), sputum smear microscopy (SSM) are conventional TB screening and diagnosis. SSM can lead to misdiagnosis due to less sensitivity (46\%), ${ }^{6}$ but it is a useful diagnostic tool especially in situations where there are large numbers of TB patients. In addition, the culture and drug susceptibility testing (DST) are used to confirm TB infection in case of negative smear and suspected to be MDR-TB, respectively. ${ }^{7}$ Given that the conventional screening and diagnosis take several weeks to complete (more than 4 weeks for culture and at least 6 weeks for DST), this can cause a delay in diagnosis and treatment for several weeks, ultimately resulting in increasing mortality and enhancing community transmission. According to a previous study, a delay of more than 21 weeks resulted in a 1.59-fold increase in mortality compared to a delay of less than 7.6 weeks (95\% CI 1.01 to 2.48 ). ${ }^{8}$

To improve the performance of TB screening and diagnosis, a rapid diagnosis method, a new technology, is required. The WHO has recommended molecular testing including Xpert MTB/RIF and loop-mediated isothermal amplification (TB-LAMP) as a diagnostic tool for TB and drug-resistant TB in suspected tuberculosis patients with signs and symptoms. The Xpert MTB/RIF is an automated cartridge-based real-time polymerase chain reaction (PCR) test by detection gene sequence of MTB and rpoB gene mutation related to rifampicin resistance. The ability of Xpert MTB/RIF is to detect both of MTB and rifampicin resistance within approximately two hours in either positive or negative sputum smear. Laboratory requirement for Xpert MTB/RIF includes stable electricity, controlled room temperature, sample temperature at 2-8 degree Celsius and regular instrument calibration. ${ }^{9}$ On the other hand, TB-LAMP is the point-of-care (POC) test, which can install at peripheral setting where microscopy is already set. TB-LAMP is temperatureindependent technique for amplifying deoxyribonucleic acid (DNA), which requires reading through ultraviolet light within 1 hour, but needs highly skilled technicians, since it is not automated like Xpert MTB/RIF. The facilities require less infrastructure and also sample can be stored at room temperature. It is highlighted that TB-LAMP can detect only MTB but not resistance TB; therefore, TB-LAMP cannot be used in high risk of MDR-TB settings ${ }^{10}$ Consequently, the molecular testing requires less laboratory equipment, small room space, less technician time, and results can be obtained in as little as 1-2 days. This can aid in improving TB case detection, allowing for prompt treatment of TB patients and ultimately reducing TB morbidity and mortality. ${ }^{11}$ It has been recommended that molecular testing should be used as an initial diagnostic test for presumptive pulmonary TB and MDR-TB. Thailand, 1 of 30 high TB burden countries, has applied the above recommendation to national policy for improving early TB case detection. ${ }^{5}$

Although molecular testing is effective and very accurate for diagnosing TB, it is still costly. Recently in Thailand, SSM with culture and DST was already covered in all health benefit schemes. However, molecular testing, ie, Xpert MTB/RIF and TB-LAMP recommended by the National TB Control Programme, ${ }^{12}$ has not been reimbursed by the Universal Health Coverage (UHC) scheme, which covered $70 \%$ of Thai populations. ${ }^{13}$ Although recently published systematic reviews provided data on the cost-effectiveness of Xpert MTB/RIF derived from a variety of settings and from several viewpoints, ${ }^{14,15}$ there is, to date, no substantial evidence evaluating the cost-effectiveness of available molecular testing, ie, Xpert MTB/RIF and TB-LAMP in Thailand. This information could be applied as supporting evidence for policymakers to make decision whether molecular testing should be included into the UHC health benefit package. Accordingly, the purpose of this study was to evaluate the cost-utility of diagnosing TB using molecular testing 
algorithms compared with SSM with culture and DST as a conventional diagnostic approach in the Thai general population suspected of having pulmonary TB.

\section{Materials and Methods}

\section{Target Population}

The Thai general population over the age of 15 years who presented with signs and symptoms related to pulmonary TB or who had an abnormal chest X-ray diagnosed and verified by a consultant chest physician and a radiologist were included in the analysis. The suspected patients were not classified as either high-risk or drug-resistant populations, as defined in the 2018 Thailand National Tuberculosis Control Program (NTP) guideline. ${ }^{12}$

\section{Model Structure}

A hybrid decision tree Markov model-based cost-utility analysis was applied to evaluate costs and outcomes of SSM with culture as well as DST and molecular testing algorithms for TB diagnosis in suspected pulmonary TB in Thailand during a lifetime period with a 1-month cycle length using a Microsoft Excel software program. At the beginning of TB diagnosis, the cohort patients would enter to the decision tree model, and it was determined that they would get the diagnostic algorithms developed from Thai NTP. ${ }^{12}$ Once the cohorts were identified as positive or active TB, they received the appropriate treatment regimens based on their drug-sensitive profile: drug sensitive TB (DS-TB), MDR-TB, or extensive drug resistant TB (XDR-TB). On the other hand, cohorts with negative TB should not undergo therapy and be considered normal population.

The Markov model was constructed based on the progression and treatment of TB disease, which was modified from Schnippel et al. ${ }^{16}$ The model health states justified initiating treatment for DS-TB with an intensive phase of 2 months followed by a continuation phase of 3-6 months. After 2 and 6 months of DS-TB treatment, the cohort who failed sputum culture conversion would undergo MDR-TB treatment. For 9 months, the MDR-TB cohort received a shorter MDR-regimen consisting of intensive phase month 1-4 and continuation phase month 5-9. Treatment failure in MDRTB was examined by sputum conversion at months 4 and 9 and transferred to XDR-TB. Treatment for XDR-TB required 20 months ( 8 months for intensive phase and 12 months for continuation phase) based on the National TB Control Programme Guideline Thailand 2018. ${ }^{12}$ If sputum conversion was not achieved after completing the treatment, the XDRTB patient was transferred to palliative care until death. At the end of treatment, if sputum culture was confirmed to be negative for DS-TB, MDR-TB, or XDR-TB, these cohorts were considered to be cured. However, the cohorts might be re-infected and returned to TB treatment again. In each health state of TB treatment, the cohort could discontinue treatment due to loss to follow-up (LTF) or death. Our model assumptions were as follows: 1) all patients would receive treatment immediately after diagnosis (no delay), 2) no patient would experience an adverse drug reaction (ADR) while taking anti-tuberculosis drugs, 3) the patients who lost to follow-up could not re-enter treatment, and 4) TB diagnosis would occur only once during the first episode (Figure 1).

This study was conducted based on a societal perspective, and the costs analyzed herein included direct medical and direct non-medical care costs that were both related to the disease and drug treatment costs associated with TB, laboratory tests for diagnosis, and formal care costs. All costs were reported in year 2021 values (Baht) adjusted by Thailand's consumer price index. ${ }^{17}$ The costs and health outcomes were discounted at a rate of $3 \%$ per annum in accordance with Thai Health Technology Assessment (HTA) guidelines. ${ }^{18}$

\section{Interventions and Comparators}

This study evaluated the following TB diagnostic algorithms developed in accordance with NTP guidance of Thailand in $2018 .^{12}$

\section{SSM + Culture/DST}

This algorithm was a comparator representing the conventional diagnostic approach currently used in Thailand. SSM was used to diagnose the whole target population. Positive SSM was advised to test with the first-line DST (FL-DST) and 

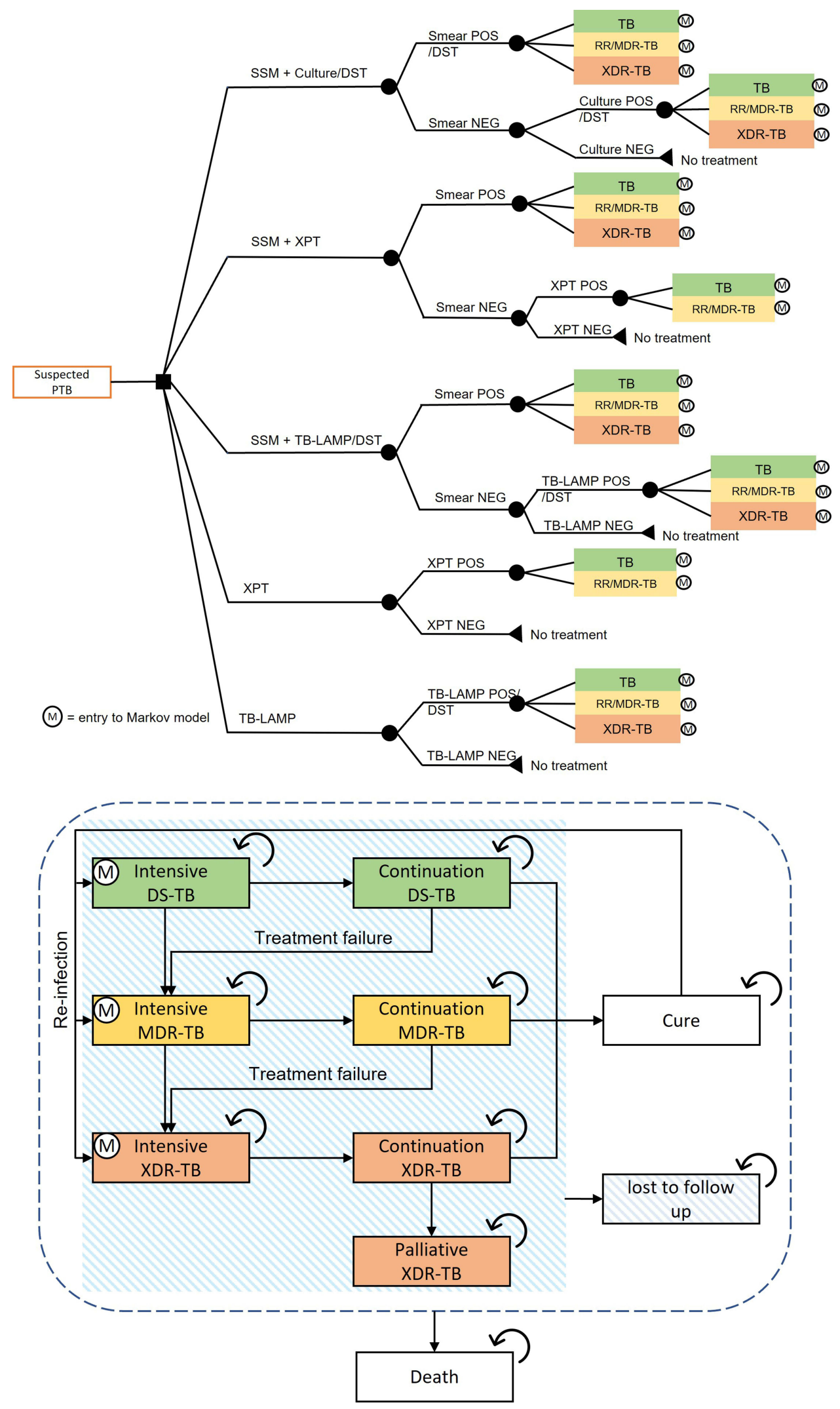

Figure I A hybrid decision tree and Markov model. Model states in five diagnostic algorithms.

Notes: The arrows represent the transition from a health state to another health state or the same health state in the next cycle.

Abbreviations: DS-TB, drug-sensitive tuberculosis; DST, drug susceptibility testing; MDR-TB, multidrug-resistant tuberculosis; NEG, negative result; POS, positive result; RR, rifampicin-resistant tuberculosis; SSM, sputum smear microscopy; XPT, Xpert MTB/RIF; XDR-TB, extensive-resistance tuberculosis. 
Table I Model Parameters

\begin{tabular}{|c|c|c|c|c|c|c|}
\hline Parameters & Distribution & Mean & SE & Alpha & Beta & References \\
\hline \multicolumn{7}{|l|}{ Transitional probability } \\
\hline $\begin{array}{l}\text { Probability of TB among suspected TB patients among abnormal } \\
\text { CXR or having clinical symptoms }\end{array}$ & Beta & 0.1723 & 0.0011 & 20,603 & 99,000 & NTIP, Thailand ${ }^{2 I}$ \\
\hline $\begin{array}{l}\text { Probability of drug-sensitive TB among diagnosed TB patients } \\
\text { (PTB) }\end{array}$ & & & & & & DRS, Thailand ${ }^{20}$ \\
\hline - Culture/DST & Beta & 0.9893 & 0.0027 & 1474 & 16 & \\
\hline - Molecular testing & Beta & 0.9825 & 0.0033 & 1517 & 27 & \\
\hline Probability of MDR-TB among diagnosed TB patients (PMDR) & & & & & & DRS, Thailand ${ }^{20}$ \\
\hline - Culture/DST & Beta & 0.0094 & 0.0026 & 14 & 1476 & \\
\hline - Molecular testing & Beta & 0.0175 & 0.0033 & 27 & 1517 & \\
\hline Probability of XDR-TB among diagnosed TB patients ( $P X D R$ ) & Beta & 0.0013 & - & - & - & $\begin{array}{l}\text { Calculation as I-pTB- } \\
\text { PMDR }\end{array}$ \\
\hline \multicolumn{7}{|l|}{ Treatment outcome } \\
\hline Probability of successful treatment & & & & & & $\begin{array}{l}\text { Global TB report } \\
2020, \mathrm{WHO}^{2}\end{array}$ \\
\hline - DS-TB & Beta & 0.8520 & 0.0012 & 72,445 & 12,584 & \\
\hline- MDR-TB & Beta & 0.5394 & 0.0158 & 58 & 392 & \\
\hline$-\mathrm{XDR}-\mathrm{TB}$ & Beta & 0.6250 & 0.1614 & 5 & 3 & \\
\hline Probability of loss to follow-up & & & & & & $\begin{array}{l}\text { Global TB report } \\
2020, \mathrm{WHO}^{2}\end{array}$ \\
\hline - DS-TB & Beta & 0.0037 & 0.0002 & 312 & 81,288 & \\
\hline- MDR-TB & Beta & 0.0104 & 0.0038 & 8.33 & 751 & \\
\hline$-\mathrm{XDR}-\mathrm{TB}$ & Beta & 0.0000 & 0.0108 & 0.008 & 8 & \\
\hline Probability of death & & & & & & $\begin{array}{l}\text { Global TB report } \\
2020, \mathrm{WHO}^{2}\end{array}$ \\
\hline - DS-TB & Beta & 0.0069 & 0.0003 & 567 & 78,227 & \\
\hline- MDR-TB & Beta & 0.0169 & 0.0051 & 13 & 694 & \\
\hline$-\mathrm{XDR}-\mathrm{TB}$ & Beta & 0.0237 & 0.0606 & 0.17 & 6 & \\
\hline Probability of reinfection after complete treatment & Beta & 0.0076 & 0.0008 & 99 & 12,930 & $\begin{array}{l}\text { Chuchottaworn C, } \\
2017^{19}\end{array}$ \\
\hline Probability of acquisition of drug-sensitive TB to MDR-TB & Beta & 0.0170 & 0.0017 & 98 & 5683 & $\begin{array}{l}\text { Global TB report } \\
2020, \mathrm{WHO}^{2}\end{array}$ \\
\hline Probability of acquisition of drug-sensitive MDR-TB to XDR-TB & Beta & 0.0190 & 0.0019 & 98 & 5068 & Menzies NA, $2012^{\prime \prime}$ \\
\hline \multicolumn{7}{|l|}{ Test performance } \\
\hline Sensitivity of SSM & Beta & 0.6100 & 0.1480 & 6.02 & 3.85 & $\mathrm{WHO}^{43}$ \\
\hline Specificity of SSM & Beta & 0.9800 & 0.0179 & 59.26 & 1.21 & $\mathrm{WHO}^{43}$ \\
\hline Sensitivity of DST & Fix & 1.0000 & - & - & - & $\begin{array}{l}\text { Assumption as gold } \\
\text { standard }\end{array}$ \\
\hline Specificity of DST & Fix & 1.0000 & - & - & - & $\begin{array}{l}\text { Assumption as gold } \\
\text { standard }\end{array}$ \\
\hline Sensitivity of liquid culture for smear-negative TB & Beta & 1.0000 & 0.0255 & 90 & 10 & $\begin{array}{l}\text { Assumption as gold } \\
\text { standard }\end{array}$ \\
\hline
\end{tabular}

(Continued) 
Table I (Continued).

\begin{tabular}{|c|c|c|c|c|c|c|}
\hline Parameters & Distribution & Mean & SE & Alpha & Beta & References \\
\hline Specificity of liquid culture for smear-negative TB & Beta & 1.0000 & 0.0255 & 90 & 10 & $\begin{array}{l}\text { Assumption as gold } \\
\text { standard }\end{array}$ \\
\hline Sensitivity of Xpert MTB/RIF followed smear negative & Beta & 0.6800 & 0.0332 & 133.86 & 62.99 & WHO9 \\
\hline Specificity of Xpert MTB/RIF followed smear negative & Beta & 0.9900 & 0.0026 & 1505.07 & 15.20 & $\mathrm{WHO}^{9}$ \\
\hline Sensitivity of Xpert MTB/RIF initial testing & Beta & 0.8800 & 0.0204 & 222.24 & 30.31 & WHO9 \\
\hline Sensitivity of Xpert MTB/RIF initial testing & Beta & 0.9900 & 0.0026 & 1505.07 & 15.20 & WHO9 \\
\hline Sensitivity of Xpert MTB/RIF for detection RR & Beta & 0.9500 & 0.0179 & 140.56 & 7.40 & WHO9 \\
\hline Sensitivity of Xpert MTB/RIF for detection RR & Beta & 0.9800 & 0.0051 & 736.91 & 15.04 & WHO9 \\
\hline Sensitivity of TB-LAMP smear negative & Beta & 0.4030 & 0.0666 & 21.47 & 31.80 & Shete PB, $2019^{22}$ \\
\hline Specificity of TB-LAMP smear negative & Beta & 0.9770 & 0.0064 & 538.79 & 12.68 & Shete PB, $2019^{22}$ \\
\hline Sensitivity of TB-LAMP all smear & Beta & 0.8030 & 0.0439 & 65.18 & 15.99 & Shete PB, $2019^{22}$ \\
\hline Specificity of TB-LAMP all smear & Beta & 0.9770 & 0.0066 & 498.07 & 11.73 & Shete PB, $2019^{22}$ \\
\hline \multicolumn{7}{|l|}{ Cost } \\
\hline \multicolumn{7}{|l|}{ Direct medical cost } \\
\hline \multicolumn{7}{|l|}{ Cost of laboratories } \\
\hline Unit cost of culture (liquid culture) & Gamma & 200 & 20 & 100 & 2 & CGD, Thailand ${ }^{24}$ \\
\hline Unit cost of DST (FL-DST and SL-DST) & Gamma & 600 & 25 & 100 & 3 & CGD, Thailand ${ }^{24}$ \\
\hline Unit cost of SSM & Gamma & 70 & 7 & 100 & I & CGD, Thailand ${ }^{24}$ \\
\hline Unit cost of CXR & Gamma & 200 & 20 & 100 & 2 & CGD, Thailand ${ }^{24}$ \\
\hline Unit cost of Xpert MTB/RIF per test & Gamma & 880 & 88 & 100 & 9 & CGD, Thailand ${ }^{24}$ \\
\hline Unit cost of TB-LAMP per test & Gamma & 880 & 88 & 100 & 9 & CGD, Thailand ${ }^{24}$ \\
\hline \multicolumn{7}{|l|}{ Cost of treatment } \\
\hline First-line drug treatment per month & & & & & & $\begin{array}{l}\text { The Median Drug } \\
\text { Prize, Thailand }\end{array}$ \\
\hline - Intensive phase: HRZE & Gamma & 482 & 48 & 100 & 5 & \\
\hline - Continuous phase: HR & Gamma & 189 & 19 & 100 & 2 & \\
\hline $\begin{array}{l}\text { MDR regimen (shorter regimen) per month } \\
\text { - Intensive phase ( } 4 \text { months): Km Mfx Pto Cfz Z E H } \\
\text { - Continuous phase ( } 5 \text { months): Mfx Cfz Z E }\end{array}$ & $\begin{array}{l}\text { Gamma } \\
\text { Gamma }\end{array}$ & $\begin{array}{l}4259 \\
3533\end{array}$ & $\begin{array}{l}426 \\
353\end{array}$ & $\begin{array}{l}100 \\
100\end{array}$ & $\begin{array}{l}43 \\
35\end{array}$ & $\begin{array}{l}\text { The Median Drug } \\
\text { Prize, Thailand }{ }^{23}\end{array}$ \\
\hline $\begin{array}{l}\text { XDR regimen (shorter regimen) per month: } 8 \mathrm{Cm} \mathrm{I2Lzd} \mathrm{20Cfz} \\
\text { 20Mfx 6Bdq } \\
\text { - Intensive phase ( } 8 \text { months) } \\
\text { - Continuous phase ( } 12 \text { months) }\end{array}$ & $\begin{array}{l}\text { Gamma } \\
\text { Gamma }\end{array}$ & $\begin{array}{l}61,488 \\
15,210\end{array}$ & $\begin{array}{l}6149 \\
152 \mid\end{array}$ & $\begin{array}{l}100 \\
100\end{array}$ & $\begin{array}{l}615 \\
152\end{array}$ & $\begin{array}{l}\text { The Median Drug } \\
\text { Prize, Thailand }\end{array}$ \\
\hline Cost of OPD service in general hospital & Gamma & 258 & 26 & 100 & 3 & $\begin{array}{l}\text { Standard Cost lists, } \\
\text { Thailand }^{25}\end{array}$ \\
\hline
\end{tabular}

(Continued) 
Table I (Continued).

\begin{tabular}{|c|c|c|c|c|c|c|}
\hline Parameters & Distribution & Mean & SE & Alpha & Beta & References \\
\hline \multicolumn{7}{|l|}{ Direct non-medical care cost } \\
\hline \multicolumn{7}{|l|}{ Before diagnosis } \\
\hline Travel cost, accommodation cost and food & Gamma & 442 & 44 & 100 & 4 & Wongrot, $2020^{26}$ \\
\hline Formal care cost & Gamma & 5 & 1 & 100 & 0.1 & Wongrot, $2020^{26}$ \\
\hline \multicolumn{7}{|l|}{ Post diagnosis } \\
\hline Travel cost, accommodation cost and food & & & & & & Wongrot, $2020^{26}$ \\
\hline - DS-TB & Gamma & 369 & 37 & 100 & 4 & \\
\hline- MDR-TB & Gamma & 839 & 84 & 100 & 8 & \\
\hline Formal care cost & & & & & & Wongrot, $2020^{26}$ \\
\hline - DS-TB & Gamma & 72 & 7 & 100 & I & \\
\hline - MDR-TB & Gamma & 0 & 0 & 0 & 0 & \\
\hline \multicolumn{7}{|l|}{ Utility } \\
\hline Ongoing DS-TB treatment & Beta & 0.6900 & 0.04 & 88 & 39 & Kittikraisak, $2012^{3}$ \\
\hline Ongoing MDR-TB treatment & Beta & 0.5100 & 0.11 & 10 & 9 & Kittikraisak, $2012^{3}$ \\
\hline Ongoing XDR-TB treatment & Beta & 0.5100 & 0.11 & 10 & 9 & Kittikraisak, $2012^{3}$ \\
\hline Treated/complete TB treatment & Beta & 0.8800 & 0.06 & 24 & 3 & Kittikraisak, $2012^{3}$ \\
\hline
\end{tabular}

Abbreviations: Bdq, bedaquiline; Cfz, clofazimine; Cm, capreomycin; CXR, chest X-ray; DST, drug susceptibility testing; DS-TB, drug-sensitive tuberculosis; E, ethambutol; FL-DST, first-line drug susceptibility testing; $\mathrm{H}$ indicates isoniazid; Km, kanamycin; Lfx, levofloxacin; Lzd, linezolid; MDR-TB, multidrug-resistant tuberculosis; Mfx, moxifloxacin; Pto, prothionamide; R, rifampicin; RR, rifampicin-resistant tuberculosis; SL-DST, second-line drug susceptibility testing; SSM, sputum smear microscopy; XDRTB, extensive-resistance tuberculosis; $Z$, pyrazinamide.

the second-line DST (SL-DST) for testing drug-resistant pattern to select the appropriate treatment regimen for the patients such as DS-TB, MDR-TB or XDR-TB treatment regimen. Although the "culture" was utilized as a confirmed diagnosis test in the negative SSM, it was not used in the positive SSM. Both FL-DST and SL-DST would then be used to categorize and assign the patients to the appropriate treatment regimen.

\section{Xpert MTB/RIF Add-on}

In smear-negative patients, the Xpert MTB/RIF interventions could be used as a secondary diagnostic or follow-on test to SSM rather than culture.

\section{Xpert MTB/RIF Initial}

Moreover, the Xpert MTB/RIF could be used as an initial diagnosis in all patients or instead of SSM. For patients receiving Xpert MTB/RIF add-on or initial, a treatment regimen that was assigned to TB patients was selected based on Xpert MTB/RIF result, which was either a DS-TB regimen or a rifampicin resistant (RR-TB) regimen.

\section{TB-LAMP Add-on}

Additionally, the TB-LAMP was selected as a follow-on test to SSM rather than culture in smear-negative patients.

\section{TB-LAMP Initial}

Alternatively, the TB-LAMP could be used as an initial diagnostic test in all patients or in place of SSM. For patients who tested positive for TB using TB-LAMP, the FL-DST and SL-DST would be utilized to determine drug resistance profile and to start the appropriate treatment regimen for patients classified as DS-TB, MDR-TB, and XDR-TB. 


\section{Model Parameters}

The probability of various variables including sensitivity, specificity of a TB diagnosis, transition probabilities between health states, costs, and health utilities were used as input parameters in the model (Table 1). This study analyzed data derived from both systematic reviews of randomized controlled trial (RCT) and meta-analysis published in Thailand or other countries.

\section{Transitional Probabilities}

Probability of treatment outcomes in terms of cure, mortality, and loss to follow-up were calculated using data from global TB report 2021 by the WHO. ${ }^{2}$ The probability of reinfection after complete treatment was calculated from Chuchottaworn et al. ${ }^{19}$ Furthermore, the probability related to TB natural parameters including the proportion of suspected TB patients with either abnormal CXR or clinical symptoms, the proportion of DS-TB, MDR-TB, or XDR-TB in each intervention was retrieved from the National Tuberculosis Information Program, Division of tuberculosis, Ministry of Public Health, Thailand. ${ }^{20,21}$ Sensitivity and specificity of diagnostic tests were obtained from the WHO and meta-analysis studies. ${ }^{9,22}$ All probabilities were calculated in unit of a month when they were linked to a cycle length at 1 month.

\section{Costs}

Direct medical and direct non-medical costs were both calculated from literature reviews and previous studies that gathered data on TB in Thailand. Direct medical costs included costs related to disease and drug treatment costs consisting of cost of anti-tuberculosis drugs: first-line drug regimen being 2HRZE/4HR, MDR-TB shorter regimen being 4Km Mfx Pto Cfz Z E H/5 Mfx Cfz Z E, and XDR-TB regimen being 8Cm 12Lzd 20Cfz 20Mfx 6Bdq, ${ }^{23}$ in addition to laboratory costs for TB screening and diagnosis (CXR, SSM, Culture, DST, Xpert MTB/RIF, TB-LAMP) and costs of monitoring (OPD charge). ${ }^{23-25}$ Direct non-medical costs including transportation costs, costs of food, hotel costs, and formal care costs were retrieved from Wongrot et al. ${ }^{26}$

\section{Utilities}

The health utility was used to calculate the quality-adjusted life years (QALYs). Data on the utilities of DS-TB, MDRTB, and cure were obtained from Kittikraisak et al. ${ }^{3}$ The utility of XDR-TB was considered to be equivalent to that of the MDR-TB health state. The health utility of patients who were classified as LTF in either DS-TB, MDR-TB, or XDR-TB health states was believed to be equivalent to the treatment received before to their loss. In addition to this, the utility of palliative care was half that of XDR-TB throughout treatment.

\section{Uncertainty Analysis}

For uncertainty analysis in treatment costs and effective parameters, which had an influence on the results of cost-effectiveness analysis, one-way sensitivity analysis and probability sensitivity analysis (PSA) were undertaken based on economic evaluation in health care practice. ${ }^{27}$ One-way sensitivity analysis was used to determine the impact on the predicted costs and outcomes of changing the value of a specified variable. The transitional probability was changed by $95 \%$ confidence interval $(\mathrm{CI})$ or $10 \%$ in the absence of $95 \% \mathrm{CI}$, cost parameters $25 \%$, and discounting rate $3 \%$. The parameters that had the greatest impact on the results were presented as a tornado diagram. We analysed the PSA using Monte Carlo simulation for 5000 iterations at random, and the parameter values underwent their distributions. The impact of uncertainty on the result of an economic evaluation was represented as a cost-effectiveness plane and a cost-effectiveness acceptability curve.

\section{Result Presentation}

This study measured outcomes in the terms of total costs, life-year gained (LYs), and QALYs, which are computed by multiplying LYs by a quality of life score (utility score). The incremental cost-effectiveness ratio (ICER) was used to describe the cost-effectiveness of healthcare interventions in cost-effectiveness analysis. The ICER is defined as the ratio of the change in costs between a conventional algorithm and molecular testing algorithms divided by the difference in effectiveness outcomes for each intervention, with a cost-effectiveness threshold of 160,000 Baht per QALY gained. ${ }^{28}$ 
Table 2 Cost-Effectiveness Results of Smear + Culture/DST Compared with Molecular Testing Algorithms for TB Diagnosis in Thailand (Baht, 202I)

\begin{tabular}{|c|c|c|c|c|c|}
\hline Costs and Outcomes & SSM + Culture/DST & $\begin{array}{c}\text { Xpert MTB/RIF } \\
\text { Add-on }\end{array}$ & Xpert MTB/RIF Initial & $\begin{array}{l}\text { TB-LAMP } \\
\text { Add-on }\end{array}$ & TB-LAMP Initial \\
\hline Total cost & 6845 & 8924 & 7010 & 7626 & 6565 \\
\hline LYs & 24.73 & 25.38 & 25.52 & 25.51 & 25.59 \\
\hline QALYs & 24.24 & 24.77 & 25.07 & 25.02 & 25.17 \\
\hline Incremental cost & & 2079 & 165 & 781 & -280 \\
\hline Incremental LYs & & 0.64 & 079 & 0.78 & 0.85 \\
\hline Incremental QALYs & & 0.53 & 0.84 & 0.79 & 0.94 \\
\hline ICER per LY gained & & 3228 & 208 & 1005 & Dominant \\
\hline ICER per QALY gained & & 3940 & 197 & 993 & Dominant \\
\hline
\end{tabular}

Notes: Dominant indicates lower cost but higher effectiveness.

Abbreviations: ICER, incremental cost-effectiveness ratio; LY, life year; QALY, quality-adjusted life year; SSM, sputum smear microscopy.

\section{Results}

\section{Cost-Utility Analysis}

This base case analysis included patients over the age of 15 years having either symptoms related to PTB or abnormal CXR, and cost-effectiveness results of each algorithm are summarized in Table 2. Without molecular testing, the total cost of SSM + Culture/DST was 6845 Baht. The total cost of Xpert MTB/RIF Add-on increased to 8924 Baht; however, molecular testing could decrease the cost of TB-LAMB Initial to 6565 Baht (4\% decrease). Overall, the total cost of molecular testing algorithm uncovered that Xpert MTB/RIF Add-on (8924 Baht) and TB-LAMP Add-on (7626 Baht) were more expensive than Xpert MTB/RIF Initial (7010 Baht) and TB-LAMP Initial (6565 Baht). The molecular testing algorithms had more favorable outcomes than those without molecular testing, thereby possibly increasing LY by 0.64 to 0.85 years and QALY by 0.53 to 0.94 years. In parallel with this, the cost-effectiveness analysis revealed that TB-LAMP Initial was dominant, indicating that TB-LAMP was less expensive and more beneficial for TB diagnosis than SSM + Culture/DST. Additionally, Xpert MTB/RIF Initial had the lowest ICER (197 Baht per QALY gained), followed by TBLAMP Add-on (993 Baht per QALY gained) and Xpert MTB/RIF Add-on (3940 Baht per QALY gained).

\section{Uncertainty Analysis}

One-way sensitivity analysis represented as tornado diagram is illustrated in Figure 2. Result of TB-LAMP Initial showed that sensitivity of TB-LAMP was more sensitive than other parameters. Furthermore, discounting rate of costs at $3 \%$, unit cost of TB-LAMP, discounting rate of outcome, and specificity of SSM were all found to be influenced the results.

Figure 3A demonstrates the cost-effectiveness plane of molecular testing alternatives compared with SSM + Culture/ DST, in which the green line represented the WTP threshold at 160,000 Baht per QALY gained in Thailand. The finding indicated that almost all simulations of TB-LAMP either initial or add-on were below the threshold. Apart from those analyses, the PSA was further executed and presented as the cost-effectiveness acceptability curve (Figure 3B). The analysis showed that of all TB diagnostic test algorithms, TB-LAMP Initial had the highest probability of being costeffective (83.4\%) at the WTP 160,000 Baht per QALY-gained.

\section{Discussion}

This study is the first to evaluate the cost-utility of molecular testing algorithms including Xpert MTB/RIF and TBLAMP compared with SSM with culture and DST in the Thai general population suspected of having pulmonary TB in Thailand. Currently, the SSM with culture and DST which have been widely used as a conventional approach, but 


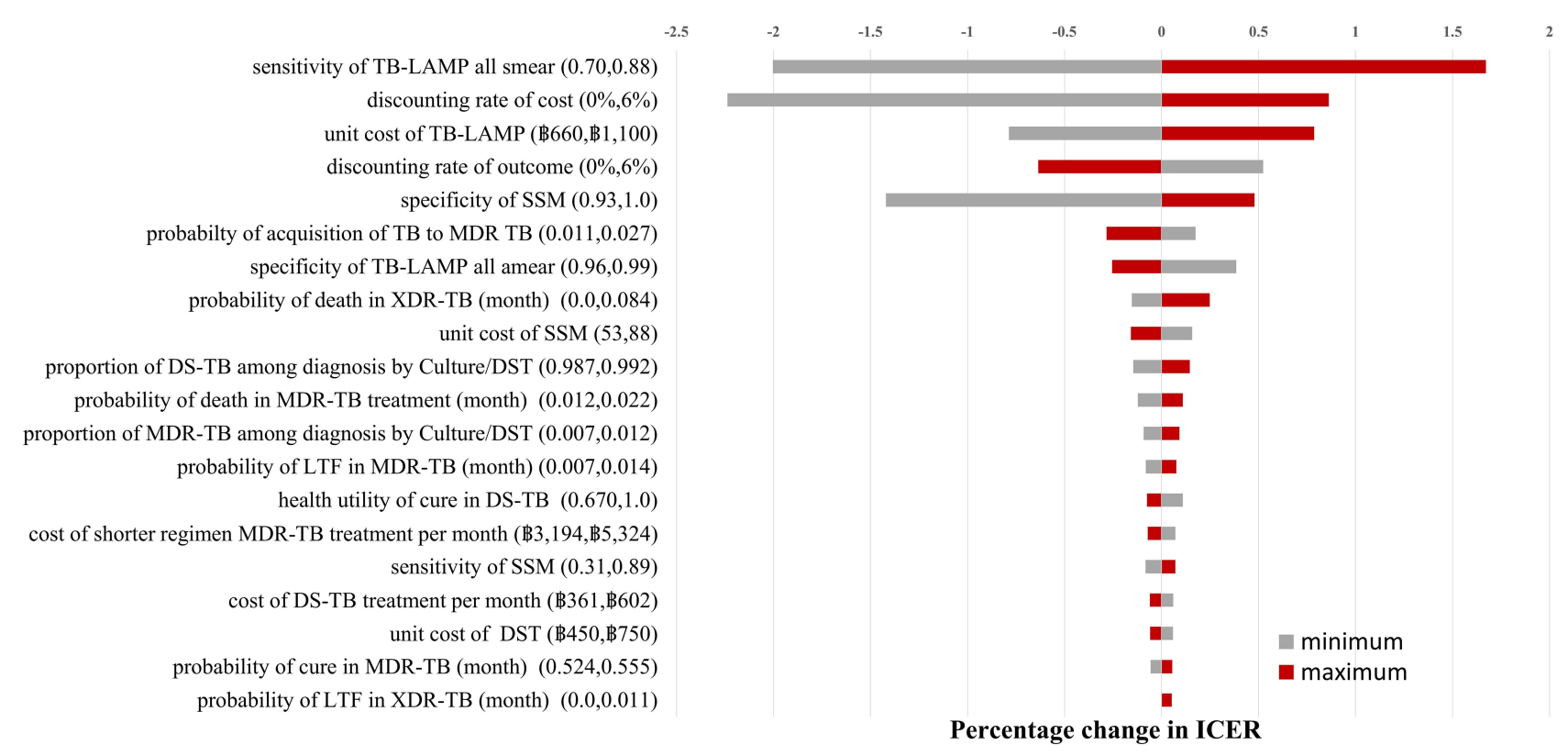

Figure 2 Tornado diagram comparing the provision of TB-LAMP Initial with SSM + Culture/DST.
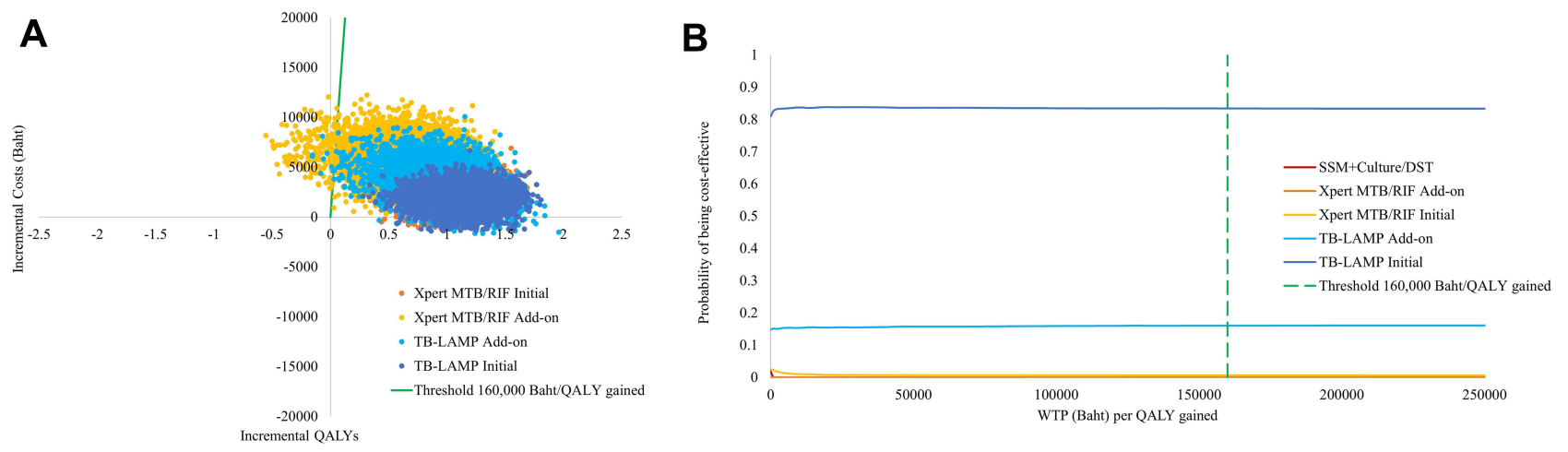

Figure 3 (A) Cost-effectiveness plane. (B) Cost-effectiveness acceptability curve.

typically takes several weeks to months to complete, thereby preventing patients from receiving therapy, ${ }^{12,15}$ has been already reimbursed in Thailand's health benefit packages, but not Xpert MTB/RIF and TB-LAMP which have been recommended by the National TB Control Programme.

The results of this study suggested that the molecular testing including Xpert MTB/RIF and TB-LAMP used as either an initial or add-on test was more cost-effective than SSM with culture and DST at the WTP of 160,000 Baht per QALY gained. Interestingly enough, providing an initial TB-LAMP test was a dominant option yielding lower cost and higher effectiveness compared with a conventional approach. ${ }^{28}$ Furthermore, the results derived from one-way sensitivity analysis unveiled that the sensitivity of TB-LAMP used as an initial test was greater than other parameters. Additionally, the finding derived from PSA suggested that of all molecular testing alternatives, providing an initial TBLAMP test would be the most cost-effective at various levels of WTP.

Our finding was consistent with several previous cost-utility analysis studies of Xpert MTB/RIF ${ }^{11,29-37}$ suggesting that Xpert MTB/RIF was more cost-effective than SSM with sputum culture. Similarly, a study by Khumsri et al ${ }^{15}$ conducted in Thailand revealed that Xpert MTB/RIF was more cost-effective than SSM. 
Nonetheless, our result differed from previous studies conducted in Malawi ${ }^{38}$ and South Africa, ${ }^{39}$ indicating that the cost-effectiveness of Xpert MTB/RIF was similar to light-emitting diodes (LED) microscopy and sputum smear. For possible reasons for conflicting results, there were a variety of methodological issues were found in our study compared to other studies. First, all data and parameters in our study were retrieved from the WHO and the National Tuberculosis Information Program, which could realistically represent the TB patients in Thailand. ${ }^{2,21}$ Second, we included all possible molecular testing algorithms including Xpert MTB/RIF and TB-LAMP currently available and ready to be implemented in Thailand for TB diagnosis. These TB diagnostic algorithms were suggested and approved by TB clinical experts and stakeholders related to TB in Thailand. Third, we used SSM with culture and DST as a comparator, which was a standard practice recommended by the National TB Control Programme. ${ }^{12}$ Last, most previous studies used a decision analysis model to simulate TB infection across the TB diagnosis algorithms, whereas we applied a hybrid decision tree and Markov model to imitate that TB could infect and re-infect individuals throughout their lives. ${ }^{19,40}$ Therefore, costs and health outcomes associated with TB diagnosis and treatment in our study could be captured realistically.

It was noted that the results of this study served as supporting evidence for the Subcommittee for the Development of the UHC benefit package under the National Health Security Office (NHSO) in determining whether molecular testing should be included in the benefit package. As a result, Xpert MTB/RIF has been covered by the UHC health insurance scheme as the rapid molecular assay for detecting TB in general population or high-risk population, ie, prisoner, household closed contact who is abnormal chest X-ray (CXR) since $2020 .^{13}$

Although our study uncovered that an initial TB-LAMP was a dominant option and more cost-effective than Xpert MTB/RIF when compared with SSM with culture and DST, the implementation of TB-LAMP in healthcare settings requires additional considerations. According to the WHO policy recommendations, the use of TB-LAMP for diagnosis of pulmonary TB still has some limitations. First, the TB-LAMP operation requires highly skilled technicians, because it is not fully automated like Xpert MTB/RIF. Subsequently, it should be concerned whether it would be economically worth setting TB-LAMP in a diagnostic service and training of the laboratory personnel. Second, the most notable limitation is the fact that TB-LAMP should not be utilized in countries with a high risk of MDR-TB, since TB-LAMP is incapable of detecting drug resistance at the time of detection and requires confirmation with DST. Third, it cannot be recommended in substitution of the Xpert MTB/RIF, because Xpert MTB/RIF has been reported to detect both TB and rifampicin resistant TB. Forth, Xpert MTB/RIF machines have already been set up at various hospitals, particularly at peripheral hospitals where TB-LAMP cannot replace. ${ }^{10}$ Last, Xpert MTB/RIF machines also have been used to diagnose non-tuberculous mycobacteria pulmonary and coronavirus infectious disease, which are likely to be useful in a pandemic situation. In light of the aforementioned reasons along with our findings, the Xpert MTB/RIF may be a viable alternative for rapid diagnostic test in countries with a high TB burden like Thailand.

Despite the important findings presented herein, there were several limitations that needed to be taken into considerations as follows. First, the model assumed that all patients received treatment, while the actual TB situation in Thailand in 2019 indicated that only $84 \%$ of the patients received treatment. For that reason, our analysis would overestimate the cost and benefit of TB diagnosis. ${ }^{2}$ Second, given that our model assumed that all patients had no adverse drug reactions (ADRs) such as hepatic dysfunction, fatigue, and vomiting, whereas in reality, most patients had symptomatic ADRs, this might result in an underestimate of cost and benefit during TB therapy. ${ }^{41}$ Third, our analysis employed static models (decision tree model and Markov model) to evaluate outcomes that do not take into account for infectious disease or TB that can be transmitted between individuals. In that context, it has been suggested that by substituting a dynamic model for the static model, we can capture additional perspectives including transmission layer, thus increasing the reliability of the results. ${ }^{42}$ Forth, our study gathered all of the parameters via a literature review; however, certain factors, such as sensitivity of the TB-LAMP parameter, were not performed in the Thailand setting. Nonetheless, an uncertainty analysis was performed to demonstrate the robustness of results. For those reasons, these restrictions should be modified for future research. 


\section{Conclusion}

This study provided supporting evidence suggesting that molecular testing for TB diagnosis including Xpert MTB/RIF and TB-LAMP was more cost-effective than SSM with culture and DST in the general population with suspected pulmonary TB in Thailand. Our research may provide valuable information for policymakers advocating for the inclusion of molecular testing as a means of rapid diagnosis in Thailand's UHC health benefit package, in accordance with the WHO and Thailand's operational strategy to eradicate TB.

\section{Ethics Approval}

Our study used data related to quality of life as well as direct medical and non-medical costs from published studies. The Institutional Review Boards (IRB) of Mahidol University approved this study (COA. No. MU-DT-PY-IRB 2019/02.0701) through the expedited review procedure. The need for informed consent for this study was waived by the ethics committees, as all data were obtained from published studies. All procedures performed in the study were in compliance with international guidelines for human research protection such as Declaration of Helsinki, the Belmont Report.

\section{Acknowledgments}

The authors would like to appreciate the Division of Tuberculosis, Department of Disease Control ministry of Public Health Thailand for support, providing epidemiological TB data, and giving guidance about data. This study was funded by the Health System Research Institute (HSRI), Thailand. The funder had no involvement with analysis, results, conclusion, or preparation of manuscript. The first author acknowledges the Thailand Science Research and Innovation under the Ministry of Higher Education, Science, Research and Innovation for providing the Royal Golden Jubilee Ph.D. scholarship for NC and UC (grant no. PHD/0104/2559).

\section{Disclosure}

The authors report no conflicts of interest in this work.

\section{References}

1. Centers for Disease Control and Prevention. Core Curriculum on Tuberculosis: What the Clinician Should Know. Division of Tuberculosis Elimination; 2013.

2. World Health Organization. Global tuberculosis report 2021. Geneva: World Health Organization; 2021.

3. Kittikraisak W, Kingkaew P, Teerawattananon Y, et al. Health related quality of life among patients with tuberculosis and HIV in Thailand. PLoS One. 2012;7(1):e29775. doi:10.1371/journal.pone.0029775

4. World Health Organization. The End TB Strategy. Geneva: World Health Organization; 2015.

5. Thailand operational plan to end tuberculosis 2017-2021. Division of Tuberculosis Department of Disease Control Ministry of Public Health Thailand; 2018. Available from: https://tbthailand.org/download/Manual/Thailand\%20Operational\%20Plan\%20To\%20End\%20\%20TB_2017_ 2021.pdf. Accessed January 20, 2022.

6. Chadha VK, Anjinappa SM, Rade K, et al. Sensitivity and specificity of screening tools and smear microscopy in active tuberculosis case finding. Indian J Tuberc. 2019;66(1):99-104. doi:10.1016/j.ijtb.2018.05.015

7. World Health Organization. National tuberculosis program guideline, Thailand. Division of Tuberculosis Department of Disease Control Ministry of Public Health Thailand; 2018.

8. Virenfeldt J, Rudolf F, Camara C, et al. Treatment delay affects clinical severity of tuberculosis: a longitudinal cohort study. BMJ Open. 2014;4(6): e004818. doi:10.1136/bmjopen-2014-004818

9. World Health Organization. Policy Update: Xpert MTB/RIF Assay for the Diagnosis of Pulmonary and Extrapulmonary TB in Adults and Children. Geneva: World Health Organization; 2017.

10. World Health Organization. The Use of Loop-Mediated Isothermal Amplification (TB-LAMP) for the Diagnosis of Pulmonary Tuberculosis. Geneva: World Health Organization; 2016.

11. Menzies NA, Cohen T, Lin HH, et al. Population health impact and cost-effectiveness of tuberculosis diagnosis with Xpert MTB/RIF: a dynamic simulation and economic evaluation. PLoS Med. 2012;9(11):e1001347. doi:10.1371/journal.pmed.1001347

12. World Health Organization. National tuberculosis control programme guideline Thailand 2018. Division of Tuberculosis Department of Disease Control Ministry of Public Health Thailand; 2018.

13. NHSO annual report year 2020. Thailand: National Health Security Office, Ministry of Public Health; 2020. Available from: https://www.nhso.go. th/. Accessed August 25, 2021.

14. Hao X, Lou H, Bai J, et al. Cost-effectiveness analysis of Xpert in detecting Mycobacterium tuberculosis: a systematic review. Int J Infect Dis. 2020;95:98-105. doi:10.1016/j.ijid.2020.03.078

15. Khumsri J, Hanvoravongchai P, Hiransuthikul N, et al. Cost-effectiveness analysis of Xpert MTB/RIF for multi-outcomes of patients with presumptive pulmonary tuberculosis in Thailand. Value Health Reg Issues. 2020;21:264-271. doi:10.1016/j.vhri.2019.09.010 
16. Schnippel K, Firnhaber C, Page-Shipp L, et al. Impact of adverse drug reactions on the incremental cost-effectiveness of bedaquiline for drug-resistant tuberculosis. Int J Tuberc Lung Dis. 2018;22(8):918-925. doi:10.5588/ijtld.17.0869

17. Trade Policy and Strategy Office. Report for consumer price index of Thailand YEAR 2021 BASE YEAR 2019. Thailand: Ministry of Commerce; August 5, 2021. Available from: http://www.price.moc.go.th/price/cpi/index_new_all.asp. Accessed January 20, 2022.

18. Guideline Development Working Group, the Medical Association of Thailand. Guideline for health technology assessment in Thailand updated edition: $2019 ; 2021$.

19. Chuchottaworn C, Roongruangpitayakul C. Drug susceptibility test and treatment outcome of recurrent pulmonary tuberculosis in Thailand. $J$ Med Assoc Thai. 2017;100:733

20. Division of Tuberculosis Department of Disease Control Ministry of Public Health Thailand. Drug resistance surveillance (DRS) 5th. supranational Thailand reference laboratory. Available from: https://srlthailand.org/DRS5TH/Enrollmentreport.php\#. Accessed July 29, 2021.

21. National Tuberculosis Information Program (NTIP). Division of tuberculosis department of disease control ministry of public health Thailand; July 29, 2021. Available from: http://tbcmthailand.ddc.moph.go.th/UIForm/Tableau/TEST_tbcm.php. Accessed July 29, 2021.

22. Shete PB, Farr K, Strnad L, et al. Diagnostic accuracy of TB-LAMP for pulmonary tuberculosis: a systematic review and meta-analysis. BMC Infect Dis. 2019;19(1):268. doi:10.1186/s12879-019-3881-y

23. Drug And Medical Supply Information Center Ministry of Public Health Thailand. The median drug prize. Available from: http://dmsic.moph.go. th/index/drugsearch/3. Accessed July 27, 2021.

24. Thai Laboratory List (TLL). The comptroller general's department, Thailand. Available from: https://mbdb.cgd.go.th/wel/searcheqpandmed.jsp\#. Accessed July 27, 2021.

25. Riewpaiboon A. Standard Cost Lists for Health Technology Assessment. Bangkok: Tana Press Co., Ltd.; 2011.

26. Wongrot P. Economic burden of out-of-pocket payment for healthcare expenditures of tuberculosis patients in Thailand [dissertation]. Mahidol University; 2020.

27. Drummond M, McGuire A. Economic Evaluation in Health Care: Merging Theory with Practice. Oxford University Press; 2001.

28. Thavorncharoensap M, Teerawattananon Y, Natanant S, et al. Estimating the willingness to pay for a quality-adjusted life year in Thailand: does the context of health gain matter? Clinicoecon Outcomes Res. 2013;5:29-36. doi:10.2147/ceor.S38062

29. Adelman M, Mcfarland D, Tsegaye M, et al. Cost-effectiveness of the World Health Organization-recommended algorithms for tuberculosis case finding at Ethiopian Human Immunodeficiency Virus (HIV) Clinics. Open Forum Infect Dis. 2016;3(suppl_1):549. doi:10.1093/ofid/ofw172.412

30. Choi HW, Miele K, Dowdy D, et al. Cost-effectiveness of Xpert ${ }^{\mathbb{R}}$ MTB/RIF for diagnosing pulmonary tuberculosis in the United States. Int J Tuberc Lung Dis. 2013;17(10):1328-1335. doi:10.5588/ijtld.13.0095

31. Langley I, Lin -H-H, Egwaga S, et al. Assessment of the patient, health system, and population effects of Xpert MTB/RIF and alternative diagnostics for tuberculosis in Tanzania: an integrated modelling approach. Lancet Glob Health. 2014;2(10):e581-e591. doi:10.1016/S2214-109X (14)70291-8

32. Pinto M, Steffen RE, Cobelens F, et al. Cost-effectiveness of the Xpert ${ }^{\circledR}$ MTB/RIF assay for tuberculosis diagnosis in Brazil. Int J Tuberc Lung Dis. 2016;20(5):611-618. doi:10.5588/ijtld.15.0455

33. Shah L, Rojas M, Mori O, et al. Cost-effectiveness of active case-finding of household contacts of pulmonary tuberculosis patients in a low HIV, tuberculosis-endemic urban area of Lima, Peru. Epidemiol Infect. 2017;145(6):1107-1117. doi:10.1017/s0950268816003186

34. Tesfaye A, Fiseha D, Assefa D, et al. Modeling the patient and health system impacts of alternative xpert ${ }^{\circledR}$ MTB/RIF algorithms for the diagnosis of pulmonary tuberculosis in Addis Ababa, Ethiopia. BMC Infect Dis. 2017;17(1):318. doi:10.1186/s12879-017-2417-6

35. Wikman-Jorgensen PE, Llenas-García J, Pérez-Porcuna TM, et al. Microscopic observation drug-susceptibility assay vs. Xpert ${ }^{\circledR}$ MTB/RIF for the diagnosis of tuberculosis in a rural African setting: a cost-utility analysis. Trop Med Int Health. 2017;22(6):734-743. doi:10.1111/tmi.12879

36. Winetsky DE, Negoescu DM, DeMarchis EH, et al. Screening and rapid molecular diagnosis of tuberculosis in prisons in Russia and Eastern Europe: a cost-effectiveness analysis. PLoS Med. 2012;9(11):e1001348. doi:10.1371/journal.pmed.1001348

37. You JHS, Lui G, Kam KM, et al. Cost-effectiveness analysis of the Xpert MTB/RIF assay for rapid diagnosis of suspected tuberculosis in an intermediate burden area. $J$ Infect. 2015;70(4):409-414. doi:10.1016/j.jinf.2014.12.015

38. Zwerling AA, Sahu M, Ngwira LG, et al. Screening for tuberculosis among adults newly diagnosed with HIV in Sub-Saharan Africa: a cost-effectiveness analysis. J Acquir Immune Defic Syndr. 2015;70(1):83-90. doi:10.1097/QAI.0000000000000712

39. Vassall A, Siapka M, Foster N, et al. Cost-effectiveness of Xpert MTB/RIF for tuberculosis diagnosis in South Africa: a real-world cost analysis and economic evaluation. Lancet Glob Health. 2017;5(7):e710-e719. doi:10.1016/S2214-109X(17)30205-X

40. Cudahy PGT, Wilson D, Cohen T. Risk factors for recurrent tuberculosis after successful treatment in a high burden setting: a cohort study. BMC Infect Dis. 2020;20(1):789. doi:10.1186/s12879-020-05515-4

41. Apichaya T, Rapin P. Symptom experience of adverse drug reaction among male and female patients with newly diagnosed pulmonary tuberculosis in Thailand. Belitung Nurs J. 2021;7(3). doi:10.33546/bnj.1337

42. Lugnér AK, Mylius SD, Wallinga J. Dynamic versus static models in cost-effectiveness analyses of anti-viral drug therapy to mitigate an influenza pandemic. Health Econ. 2010;19(5):518-531. doi:10.1002/hec.1485

43. World Health Organization. Systematic Screening for Active Tuberculosis: An Operation Guide. Geneva: World Health Organization; 2015.

ClinicoEconomics and Outcomes Research

Dovepress

\section{Publish your work in this journal}

ClinicoEconomics and Outcomes Research is an international, peer-reviewed open-access journal focusing on Health Technology Assessment, Pharmacoeconomics and Outcomes Research in the areas of diagnosis, medical devices, and clinical, surgical and pharmacological intervention. The economic impact of health policy and health systems organization also constitute important areas of coverage. The manuscript management system is completely online and includes a very quick and fair peer-review system, which is all easy to use. Visit http://www.dovepress.com/testimonials.php to read real quotes from published authors.

Submit your manuscript here: https://www.dovepress.com/clinicoeconomics-and-outcomes-research-journal 